\title{
Komplementäre Korrespondenz
}

\author{
Heidegger und Heisenberg zur Frage der Technik
}

\author{
Christina Vagt
}

Complementary Correspondence. Heidegger and Heisenberg on the Question Concerning Technology

\begin{abstract}
The essay deals with the brief yet significant correspondence between Martin Heidegger and Werner Heisenberg throughout the year 1953 that culminated in Heidegger's lecture "Die Frage nach der Technik". The letters and Heidegger's accompanying thoughts about the production of scientific evidence by means of media technology and mathematics provides a missing link between the genesis of Heidegger's own philosophy of technology and the history of modern physics. The correspondence indicates the struggle of both philosopher and physicist to understand each other but also Heidegger's awareness for the necessity of an interdisciplinary discourse on technology within the nuclear era. One of Heisenberg's letters worked through by Heidegger with crayons is published here for the first time.
\end{abstract}

Keywords: philosophy, 1953, modern physics, nuclear power, handwriting

Schlüsse/wörter: Philosophie, 1953, Moderne Physik, Atomkraft, Handschrift

Im November 1953 veranstaltete die Bayerische Akademie der Schönen Künste eine Vortragsreihe unter dem Titel Die Künste im technischen Zeitalter. Unter den Vortragenden, die an vier Tagen das Auditorium Maximum der Technischen Hochschule München füllten, befanden sich auch Martin Heidegger und Werner Heisenberg. Für Heidegger war nicht nur die Tagung an sich, sondern auch der eigene Vortrag von besonderer Bedeutung. Es war der erste größere Auftritt als Professor nach Aufhebung des Lehrverbots. Erst durch die Emeritierung am 26. September 1951 durch die Universität Freiburg erhielt Heidegger seine Rechte als Professor zurück und durfte wieder Vorlesungen halten - die aus seiner Sicht entscheidende philosophische Arbeit.

Ebenfalls im Jahr 1953 entfachte die Erstveröffentlichung der Einführung in die Metaphysik eine bundesweite Debatte über Heideggers nationalsozialistisches Engagement, nachdem Jürgen Habermas diese Schrift, die die bereits im Sommersemester 1935 gehaltene Vorlesung enthält, kritisch in der 
Frankfurter Allgemeinen Zeitung rezensiert hatte (Habermas 1953, Heidegger 1998, vgl. Heidegger, G. 2005288 f., Dämmerling 2003: 366 f., Carson 2010a: 497 f.).

Heidegger, das zeigt seine Korrespondenz mit Heisenberg, bemühte sich im Vorfeld der Tagung intensiv um den Beitrag des Quantenphysikers. Heisenberg war im Gegensatz zu Heidegger in den frühen 1950er Jahren ein hoch dekorierter und viel beschäftigter Mann, und so gestaltete sich der „Jahrhundert"-Dialog zwischen den beiden schon logistisch eher umständlich (Hempel 1990: 9).

Der Briefwechsel von 1953 bezeugt Heideggers großes Interesse an einem Austausch mit Heisenberg, verdeutlicht aber auch die Schwierigkeiten, den Quantenphysiker in die Gespräche zwischen Philosophen, Religionswissenschaftlern, Technikhistorikern und Künstlern so einzubeziehen, wie Heidegger es für nötig hielt. Er bat Heisenberg in einem ersten Brief vom 18. März 1953 um ein persönliches Treffen. ${ }^{1}$

Diese bisher unveröffentlichten Briefe und die dazu gehörigen handschriftlichen Notizen Heideggers in seinem Nachlass (im Folgenden „Heisenberg-Konvolut“ genannt) zeugen darüber hinaus von der Genese des heideggerschen Technikbegriffs innerhalb einer fast zwanzig Jahre währenden Auseinandersetzung mit der Quantenphysik. Zumindest geben die Fundstücke Anlass, den kanonisch gewordenen Vortrag Die Frage nach der Technik, den Heidegger schließlich auf der Tagung in München hielt, auch in Beziehung zu einer zunehmend technisch bedingten Ontologie innerhalb der Physik zu setzen.

Dass der quantenphysikalische Hintergrund der Technikfrage der Heideggerforschung so lange entgangen ist, mag daran liegen, dass Heideggers Briefe jahrzehntelang unbemerkt im Heisenberg-Archiv in München überdauert haben und auch die entsprechenden Konvolute im Deutschen Literaturarchiv Marbach, die Heideggers Überlegungen und Lektüren zur „Unbestimmtheitsrelation“ und „Kausalitätsdebatte“ enthalten, bisher nicht veröffentlicht wurden.

Im Zuge der Recherchen zu ihrer Heisenbergbiografie ist die Wissenschaftshistorikerin Cathryn Carson in dessen Münchner Nachlass auf den Briefwechsel mit Heidegger gestoßen (Carson 2010a: 493, 2010b). Generell sind die naturwissenschaftlichen Bezüge Heideggers bisher eher innerhalb der Wissenschaftsgeschichte der Physik und der Medienwissenschaft zum Gegenstand der Forschung geworden, wie etwa die Veröffentlichungen von Erich Hörl zu Heidegger und der Kybernetik zeigen (Hörl 2004).

Die drei Briefe Heideggers aus dem Jahre 1953 und Heisenbergs Antworten sowie die lose Sammlung aus handschriftlichen Notizzetteln, ausgeschnittenen Zeitungsartikeln, Vortragsentwürfen und Typoskrypten aus derselben Zeit ergeben in sich keinen geschlossenen Zusammenhang, werden 
aber vielleicht noch Eingang in die dritte Abteilung der Gesamtausgabe finden, wie es etwa im Fall des 2009 erschienenen und von Claudius Strube und Friedrich-Wilhelm von Herrmann hervorragend edierten Bandes Leitgedanken zur Entstehung der Metaphysik, der neuzeitlichen Wissenschaft und der modernen Technik gelungen ist.

Im Bezug auf das Heisenberg-Konvolut wäre auch eine teilweise Faksimilierung wünschenswert. Denn Heideggers farbige Unterstreichungen in den eigenen Notizen, in den versammelten wissenschaftlichen Abhandlungen des Physikers und populärwissenschaftlichen Zeitungsartikeln zu Fragen von Zeitparadoxien, Relativitäts- und Quantenphysik und vor allem auch zur bundesdeutschen Debatte rund um Atom- und Reaktortechnik lassen eine Materialität seiner philosophischen Arbeit erkennen, die noch nicht Gegenstand der Forschung war.

\section{Korrespondenz}

In einem zweiten Brief vom 9. Juni 1953 bat Heidegger Heisenberg erneut um ein persönliches Treffen, nachdem die ersten Gesprächsrunden mit den Tagungsteilnehmern offenbar nicht zufriedenstellend verlaufen waren. Daraufhin versprach Heisenberg im Juli nach München zu kommen, um auch die übrigen Teilnehmer zu treffen. ${ }^{2}$

Im August hielt Heidegger einen „vorbereitenden Vortrag im kleinen Kreis" mit dem Titel Wissenschaft und Besinnung, den er im folgenden Jahr zusammen mit Die Frage nach der Technik unter dem Titel Vorträge und Aufsätze bei Günter Neske in Pfullingen veröffentlichte (Heidegger 2004b [1954]). Er ließ Heisenberg das Manuskript zukommen, woraufhin dieser einen ersten eigenen Entwurf an die Münchner Runde schickte.

Trotz der Verständigungschwierigkeiten zwischen Physiker und Philosoph, insbesondere was die Bestimmungen von Mensch und Natur betraf, setzten sich beide intensiv mit den Fragen des anderen auseinander. Heisenbergs Stellungsnahme zu Wissenschaft und Besinnung vom 3. Oktober 1953 (vgl. Abb. 1), wurde von Heidegger gründlich bearbeitet und mit farbigen Unterstreichungen und Anmerkungen versehen. ${ }^{3}$

Heisenberg stieß sich hier insbesondere am „unscheinbaren Sachverhalt“, um den Heideggers Vortrag kreist: „Die Physik kann als Physik über die Physik keine Aussagen machen. Alle Aussagen der Physik sprechen physikalisch.“ (Heidegger 2004b [1954]: 61). Allerdings ist das Sprechen der Physik keine Unzulänglichkeit physikalischer oder naturwissenschaftlicher Forschung, sondern betrifft die Konstitution aller Wissenschaften. Zum Wesen der Wissenschaften gehörte für Heidegger eine ontologische Blindheit, das Nicht-Reflektieren der eigenen Möglichkeitsbedingungen: „Das in den 
a gömingen 3.10,13.

tich kn kidegpu:

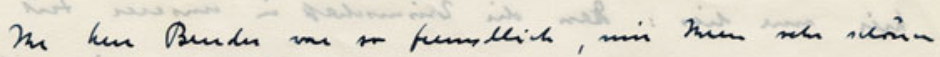

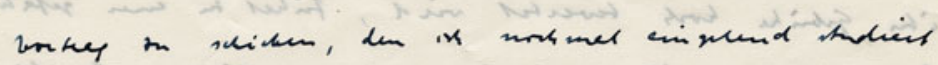

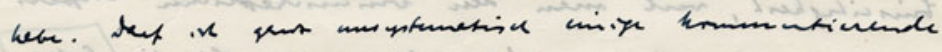

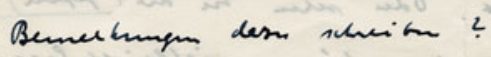

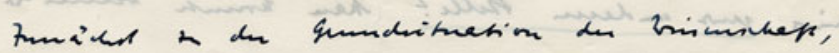

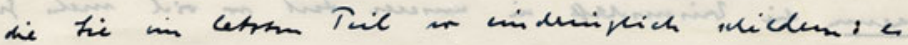

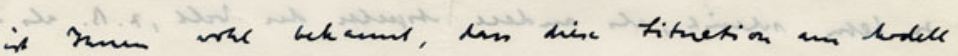

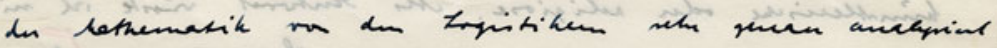

man it. Lillent wethe is perffe, ine, Therini du

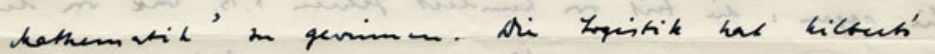

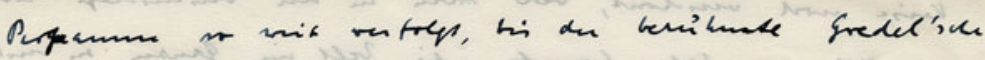

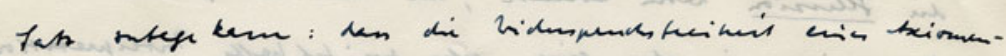

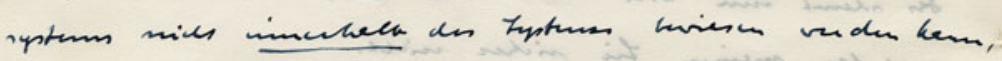

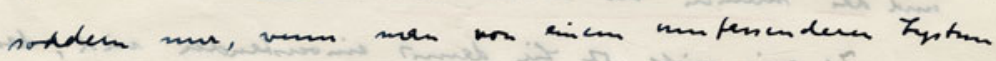

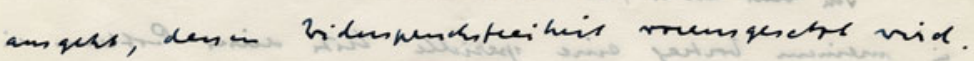

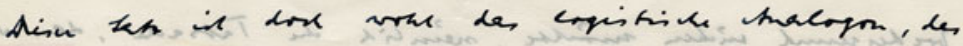

the Formulieumgn aceirter.

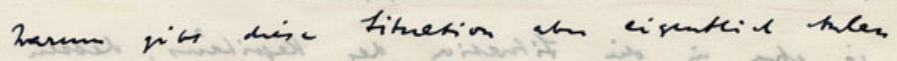

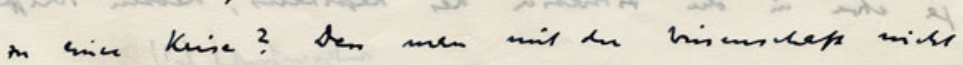

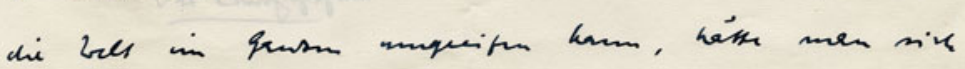

Abb. 1 Brief von Heisenberg an Heidegger vom 3. Oktober 1953, Unterstreichungen und Anmerkungen von Heidegger ( $\mathrm{MHN}$ ).

Wissenschaften jeweils Unumgängliche: die Natur, der Mensch, die Geschichte, die Sprache, ist als dieses Unumgängliche für die Wissenschaften und durch sie unzugänglich." (ebd.: 62, Hervorhebung im Original) Heisenberg verstand das philosophische Problem oder den unscheinbaren 
b

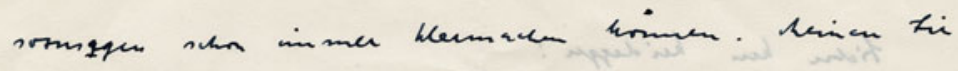

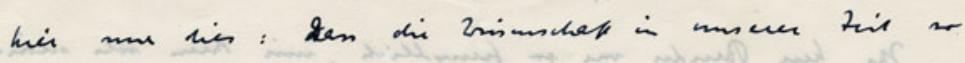

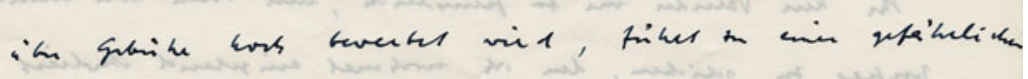

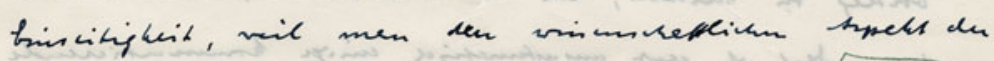

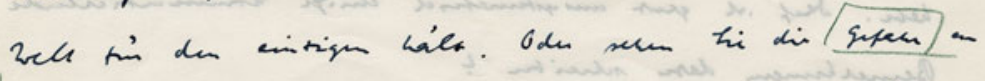

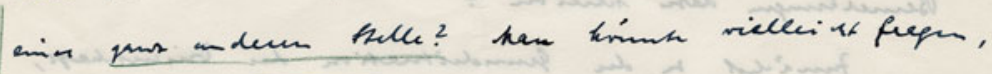

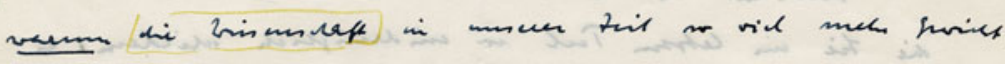

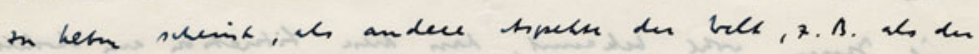

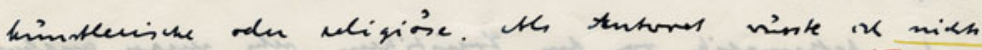

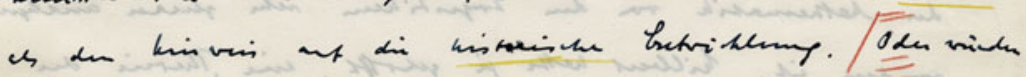

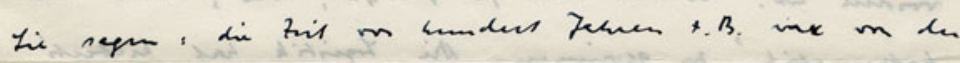

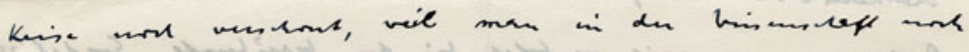

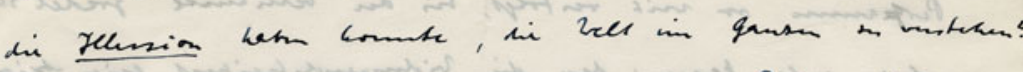

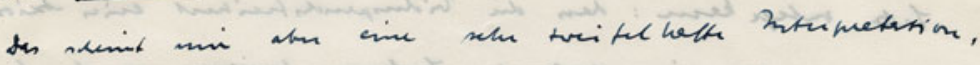
and dar meinim hi sikes wilk.

Tol min mill, is tie demint cimventender mix, den is

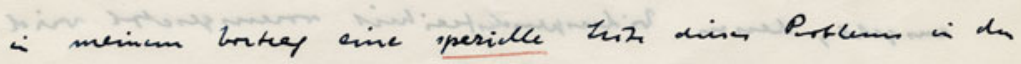

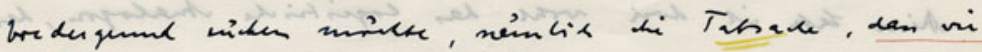

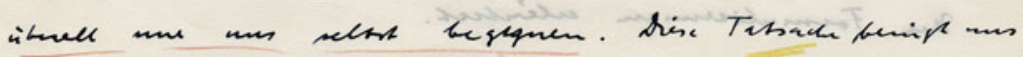

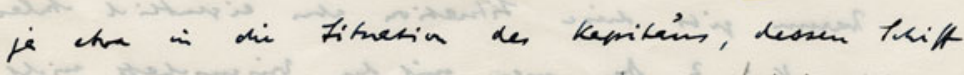
aingugafinat 102

Abb. 1 Fortsetzung Brief Heisenberg

Sachverhalt nicht und antwortete auf Heideggers Behauptung metamathematisch (vgl. Abb. 1a):

Zunächst zu der Grundsituation der Wissenschaft, die Sie im letzten Teil so eindringlich schildern: es ist Ihnen wohl bekannt, dass diese Situation am Modell der Mathematik von den Logistikern sehr genau analysiert worden ist. Hilbert hatte ja gehofft, eine ,Theorie der Mathematik zu gewinnen. Die Logistik hat Hilbert's [sic] Programm so nicht verfolgt, bis der berühmte Goedel'sche Satz 
C

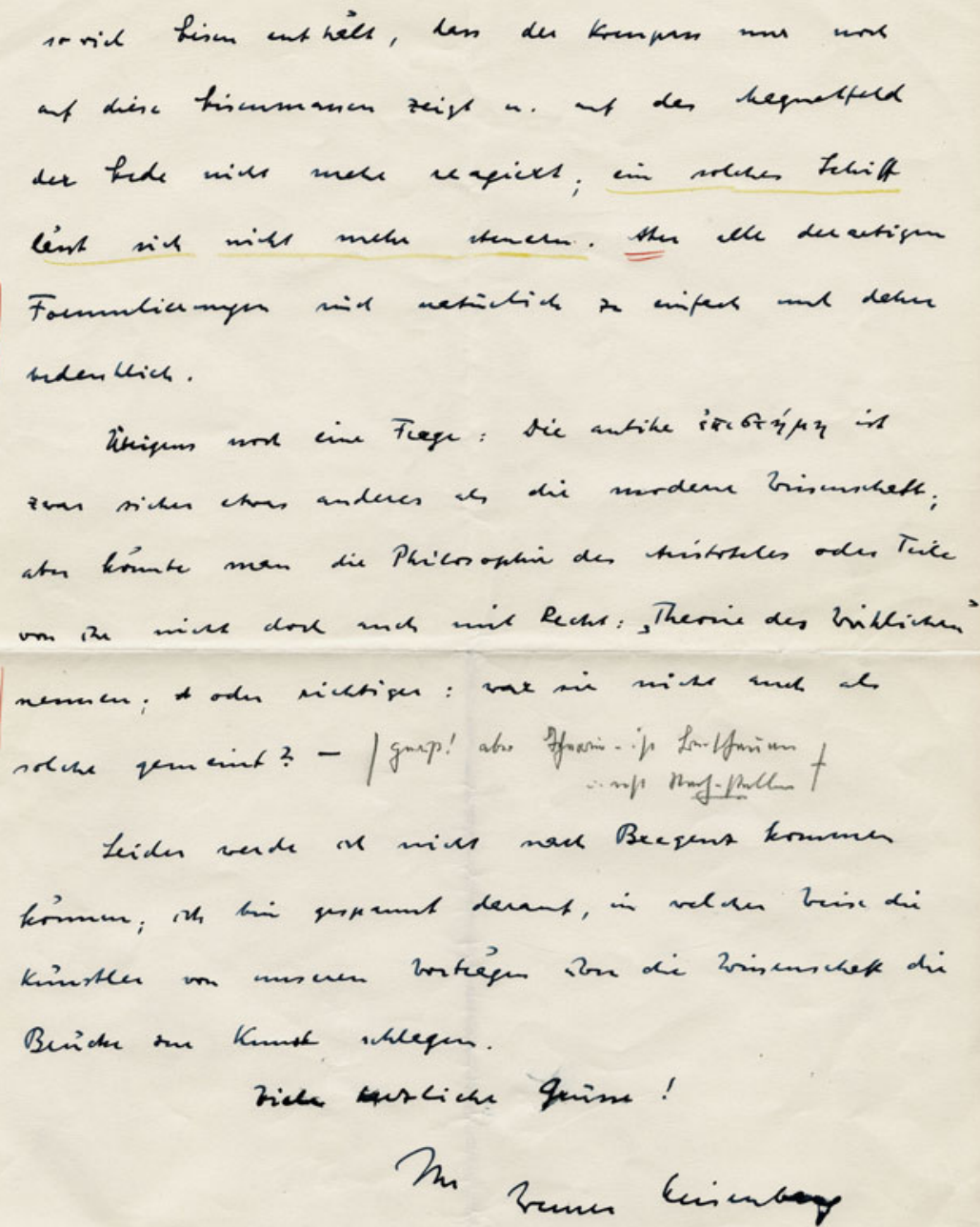

Abb. 1 Fortsetzung II, Brief Heisenberg

zutage kam: dass die Widerspruchsfreiheit eines Axiomensystems nicht innerhalb des Systems bewiesen werden kann, sondern nur, wenn man von einem umfassenderen System ausgeht, dessen Widerspruchsfreiheit vorausgesetzt wird. Dieser Satz ist doch wohl das logistische Analogon, das Ihre Formulierungen erläutert. ${ }^{4}$

Für Heisenberg ist die grundsätzliche Beschränkung eines wissenschaftlichen Systems bereits mit Kurt Gödels Entscheidungstheorem von 1931 geklärt. Die 
Widerspruchsfreiheit eines axiomatischen Systems, wie zum Beispiel der Arithmetik kann demzufolge nicht innerhalb des Systems bewiesen werden. Das zentrale Argument von Heideggers Wissenschaft und Besinnung wäre demzufolge nichts anderes als die philosophische (und verspätete) Einsicht in die Konsequenzen des mathematischen Grundlagenstreits nach dem Ersten Weltkrieg, den Gödels Satz formal beendet hatte. Heidegger, durchaus vertraut mit der Materie, wies die Analogisierung des eigenen Denkens mit der mathematischen Logik, der "Logistik“ in seiner Antwort vom 8. November 1953 entsprechend zurück:

Der Gödel'sche Satz enthält eine gewisse Analogie zu meinen Ausführungen über den ,unscheinbaren Sachverhalt'. Aber was er meint, betrifft doch nur die Beziehung eines Systems zu einem umfassenden und prinzipiell gleichartigen. Dagegen meine ich eine im Wesen andere Dimension, in die jede Wissenschaft gehört, die sie aber niemals selber erreicht. Die ,Logistik ${ }^{`}$ z.B. gelangt noch nicht und nie in die Dimension der Philosophie, sondern zieht die ,Logik' ins Mathematische ab. ${ }^{5}$

In den 1950er Jahren war der theoretische Siegeszug der gödelschen Auffassung, "that numbers can represent any kind of structure", in Form von Computern noch nicht abzusehen (Nagel/Newman 2001). Zwar spricht Heisenberg in Bezug auf die Grenzen der Beobachtung und die disparate Situation einander ausschließender Naturgesetze innerhalb der Physik von einer Situation des „zunehmenden Sichabfindens“ des Physikers (Heisenberg 1956 [1953]: 43). Doch statt die Natur „an sich“ zu beobachten, ist der Gegenstand der Forschung nun „die der menschlichen Fragestellung ausgesetzte Natur, und insofern begegnet der Mensch auch hier wieder sich selbst" (ebd.: 42).

Grundsätzlich, so der Heisenberg-Biograph Georg Schiemann, war auch diese Erkenntnis für Heisenberg Naturerkenntnis: Auch die Beherrschung der Natur gerate nur dann an ihre Grenzen, wenn sie sich mit dem Wirklichkeitsverständnis der religiösen und kulturellen Welt nicht mehr zur Deckung bringen lasse (Schiemann 2008: 122). Es sei, so Heisenberg in seinem Brief an Heidegger, wohl eher an der „religiöse[n] und kulturelle[n] Welt“, sich den neuen Naturerkenntnissen anzupassen als umgekehrt (siehe Abb. 1b):

Man könnte vielleicht fragen, warum die Wissenschaft in unserer Zeit so viel mehr Gewicht zu haben scheint, als andere Aspekte der Welt, z. B. als die künstlerische oder die religiöse. Als Antwort wüsste ich nichts als den Hinweis auf die historische Entwicklung. ${ }^{6}$

Die Quantenmechanik markierte auch für Heidegger den Höhepunkt der Mathematisierung der Naturwissenschaften und der Vergegenständlichung und Objektivierung der Natur. Da nach Heideggers Verständnis die physikalische Theorie hinter diesen Grad mathematischer Konstitution auf wissenschaftlichem Wege nicht zurück kann, muss sie ihre Gegenstände mathematisch denken. Das unterscheidet sie zunächst von dem, was Heidegger 
in der griechischen theoria als gegeben ansah: „Die griechische Wissenschaft ist eine Abwandlung der theoria, aber sie ist niemals ,Theorie' im modernen Sinn. Voraussetzung dafür wäre, daß sie das Seiende als Gegenstand zeigte. Gerade dazu kommt es nicht."7

Als Sohn eines Altphilologen durchaus mit antiker Theorie vertraut, hatte Heisenberg in seinem Brief vom 3. Oktober nach Aristoteles gefragt (siehe Abb. 1c):

Übrigens noch eine Frage: Die antike episteme [griech. im Original, C. V.] ist zwar sicher etwas anderes als die moderne Wissenschaft, aber könnte man die Philosophie des Aristoteles oder Teile von ihr nicht doch auch mit Recht: ,Theorie des Wirklichen nennen; oder richtiger: war sie nicht auch als solche gemeint? ${ }^{8}$

Die Frage schien Heidegger nachdenklich zu stimmen; er notierte sich eine stumme Antwort mit Fragezeichen auf Heisenbergs Schreiben (siehe Abb. 1c): „Gewiß! - aber Theoria ist Bestaunen (?) und nicht Nachstellen. " ${ }^{9}$ Es mag sich bei diesem Fragezeichen lediglich um eine philologische Unsicherheit handeln, darüber, ob sich theoria tatsächlich mit „Bestaunen“ übersetzen ließe. Aber vielleicht artikuliert sich hier in der Handschrift ein Zweifel Heideggers daran, was die positive Bestimmung der aristotelischen theoria und insbesondere ihr unverstelltes Verhältnis zur physis betrifft; ein Zweifel, der bereits Heideggers späte Sprachphilosophie antizipieren würde. Aber im Antwortschreiben an Heisenberg findet sich davon nichts, hier erscheint die Differenz zwischen antiker theoria und moderner, nach Naturbeherrschung strebender Wissenschaft und Technik wieder unverbrüchlich. ${ }^{10}$

Mit der Rede vom „unscheinbaren Sachverhalt" aus Wissenschaft und Besinnung nimmt Heidegger das aktuelle Grundlagenproblem der Physik, die Unvereinbarkeit von Relativitäts- und Quantenthoerie, zum Anlass, über die Grundlagenproblematik der abendländisch-rationalen Wissenschaft als solcher zu sprechen. Der Riss zwischen Relativitäts- und Quantentheorie wäre demnach ein Symptom einer ontologisch tieferligenden Grundlagenkrise der Wissenschaft, welche sich nicht durch den Aufweis prinzipieller Systemgrenzen auflösen lasse, noch würde er „durch das Erzählen der Wissenschaftsgeschichte“ zugänglich. Das grundlegende Problem der Wissenschaften ist für Heidegger wissenschaftlich schlicht nicht lösbar: „Der unscheinbare Sachverhalt verbirgt sich in den Wissenschaften. Aber er liegt nicht in ihnen wie der Apfel im Korb. Wir müssen daher sagen: die Wissenschaften ruhen ihrerseits im unscheinbaren Sachverhalt wie der Fluß im Quell." (Heidegger 2004b [1954]: 63).

Die Grundlagenkrise, die sich mit der Quantenphysik manifestiert hatte, wurde von Heidegger im Münchner Vortrag schließlich auf die antike Trennung von physis und techné zurückgeführt, die angesichts neuer physikalischer Technologien in ungekanntem und unbeherrschbarem Ausmaß ontologisch nicht länger aufrecht zu halten war. Natur und Technik seien immer schon 
miteinander verschränkt. Dieser Sachverhalt sei nicht neu, historisiert Heidegger mit Hinweis auf Descartes' rationale Methode. Er wäre allerdings erst mit der Physik des 20. Jahrhunderts ontologisch auffällig geworden. Für Heidegger betraf dann auch die Grundlagenkrise und Beunruhigung der Physik lediglich ihre Grundbegriffe, die sich nicht mehr mit der Mathematik deckten - die Wissenschaft als solche gehe jedoch sicherer denn je ihren Gang (ebd.: 62). Unanschaulichkeit war nach Heidegger für die moderne Physik kein Problem, solange sich die Natur in irgendeiner messbaren Art und Weise meldet:

Auch dort, wo die Theorie aus Wesensgründen wie in der modernen Atomphysik notwendig unanschaulich wird, ist sie darauf angewiesen, daß sich die Atome für eine sinnliche Wahrnehmung herausstellen, mag dieses Sich-zeigen der Elementarteilchen auch auf einem sehr indirekten und technisch vielfältig vermittelten Wege geschehen (vgl. Wilsonkammer, Geigerzähler, Freiballonflüge zur Feststellung der Mesonen). (ebd.: 58)

In der Evidenzproduktion der Mikrophysik sah Heidegger die doppelte Bindung des Wissens an Natur und Technik. Das theoretische Gebäude der Physik bleibe an die Vorgängigkeit der zu untersuchenden Natur gebunden, gleichzeitig müsse sie es sich als systematische Wissenschaft zur Aufgabe machen, ihren Gegenstandsbezirk - die Natur - in Formeln zu rationalisieren und so zu vereinheitlichen:

Auch für die moderne Physik gilt es, diejenigen elementaren Gegenstände sicherzustellen, aus denen alle anderen Gegenstände des ganzen Gebietes bestehen. Auch das Vorstellen der modernen Physik bleibt darauf abgestellt, ,eine einzige Grundgleichung anschreiben zu können, aus der die Eigenschaften aller Elementarteilchen und damit das Verhalten der Materie überhaupt folgt' (Heisenberg, Die gegenwärtigen Grundprobleme der Atomphysik. Vgl. Wandlungen in den Grundlagen der Naturwissenschaft, 8. Auflage, 1948 [sic], S. 98.). (ebd.: 56 f.)

In dem von Heidegger zitierten Artikel schrieb Heisenberg unter anderem über die Entdeckung von Positronen und Mesonen und gab sich noch zuversichtlich, dass die Physik schon bald auf eine die Quantenmechanik und die Allgemeine Relativitätstheorie vereinigende Grundgleichung stoßen würde. Die Zerrissenheit der Physik versuchte er mit einem Satz des Demokrit zu versöhnen: So wie Tragödie und Komödie mit denselben Buchstaben niedergeschrieben würden, so könnten auch dieselben Atome sehr verschiedene Geschehnisse in der Welt hervorbringen. Auch wenn es sich bei der Atomtheorie nicht mehr im klassischen Sinne um eine materialistische Theorie handele, sei es „wichtig, daß wir die Schrift der Atome ganz verstehen, denn diese Schrift ist nicht vom Menschen erdacht worden; sie bedeutet mehr" (Heisenberg 1949: 101).

Für Heisenberg handelte es sich um ein Problem der Entzifferung, denn für ihn war grundsätzlich die Natur als Natur lesbar. Heidegger dagegen schien sich nicht ganz darüber im Klaren zu sein, womit er es bei den neuen Detektoren und Atomschriften $\mathrm{zu}$ tun hatte. In dem unveröffentlichten 
Konvolut zu Heisenberg finden sich einige Seiten handschriftlicher Vorarbeiten aus dem Jahre 1953 unter dem Titel „Zu Heisenberg“, in denen er das Verhältnis von Physik und Technik verhandelte und einige Fragezeichen setzte:

Was grundsätzlich physikalisch als Ortsbestimmung und Impulsbestimmung nötig bleibt, stößt hinsichtlich der Ausführung sowohl von Seiten der sich herausstellenden Mikro-Natur, als auch von Seiten der experimentellen Apparatur an eine Grenze - insofern die sich herausstellende Natur zu folgender sie verwindenden (?) Apparatur (sich) nicht mehr zugleich nach Ort und Bewegungsimpuls bestimmen lässt. Die Subjektivität des sicherstellenden Subjekts greift durch die von ihm benötigte Apparatur (massiver - einmalig, kausal, effizient) in die Objektivität des sich herausstellenden Objektes ein, diese entzieht sich anders und mehr noch als bisher der vermeintlich einen Sicherstellung (der Natur an sich). Die experimentierende Subjektivität ist jetzt ausdrücklich in die ungeschmückte (?) Objektivität mit eingebaut, „strukturiert“-. die [sic] Subjektivität gehört jetzt ausdrücklicher als bisher, nämlich auch hinsichtlich der experimentellen Apparatur zur Objektivität. Zur Objektivität gehört der ausdrückliche Einbau der Subjektivität. Die Sub.-Obj.Beziehung ist jetzt eine noch massivere als bisher - sie ist radikalisiert und darum ferner einer möglichen Verwindung denn je zuvor - mit Rücksicht auf sie und für das Denken die Unzugänglichkeit des Unumgänglichen noch deutlicher aufweisbar. ${ }^{11}$

Bei der Kopenhagener Deutung der Quantenmechanik handelte es sich Heidegger zufolge gerade nicht um die Auflösung der Trennung von Subjekt und Objekt, sondern um die Entbergung ihrer ontologischen Verschränkung, die Enthüllung eines komplementären Verhältnisses. Noch nie stand der unscheinbare Sachverhalt so deutlich vor ihm wie in den Evidenzmaschinen der Mikrophysik. Ungeklärt war für ihn zu diesem Zeitpunkt noch, ob diese Apparaturen letztendlich Natur ,verwinden', und das hieße zur Sprache bringen, ob die Maschinen also zum ,Unterwegssein der Natur , gehören, was einer ontologischen Faltung gleichkäme, womit das Wesen der Physik die Technik wäre. Besagtem Konvolut von 1953 liegen noch einige Blätter mit „Fragen an Heisenberg“ bei. Eine von ihnen lautet: „Ist die Physik als s[olche] in einem wesenhaften Sinn gerade die ,Technik' und nur diese?"12

\section{Die Frage nach der Technik}

Das Heidegger-Konvolut enthält Das Naturbild der Physik, Heisenbergs Vortragsentwurf für die Münchner Tagung von 1953 (Heisenberg 1956 [1953]). Auch hier finden sich einige Unterstreichungen Heideggers, teilweise in Farbe, Anmerkungen und Fragen, die in der Frage nach der Technik wieder auftauchen. Heisenberg schildert das Dilemma einer sich ins Symbolische der Mathematik „verflüchtigenden“ physikalischen Realität:

Die Frage, ob diese Teilchen „an sich“ in Raum und Zeit existieren, kann in dieser Form also nicht mehr gestellt werden, da wir stets nur über die Vorgänge sprechen können, die sich abspielen, wenn durch die Wechselwirkung des Elementarteilchens mit irgendwelchen anderen physikalischen Systemen, z. B. den 
Meßapparaten, das Verhalten des Teilchens erschlossen werden soll. Die Vorstellung von der objektiven Realität der Elementarteilchen hat sich also in einer merkwürdigen Weise verflüchtigt, nicht in den Nebel irgendeiner neuen, unklaren oder noch unverstandenen Wirklichkeitsvorstellung, sondern in die durchsichtige Klarheit einer Mathematik, die nicht mehr das Verhalten des Elementarteilchens sondern unsere Kenntnis dieses Verhaltens darstellt. Der Atomphysiker hat sich damit abfinden müssen, daß seine Wissenschaft nur ein Glied ist in der endlosen Kette der Auseinandersetzungen des Menschen mit der Natur, daß sie aber nicht einfach von der Natur ,an sich" sprechen kann. ${ }^{13}$

Heidegger notierte sich dazu am Rand eine Frage: „Was liegt in diesem Abfinden? Wo befindet sich der Physiker jetzt?"14 In Die Frage nach der Technik schrieb er dann, dass die moderne Physik die Natur als „vorausberechenbaren Zusammenhang von Kräften“ stelle und sie zum „Bestand“ mache. Im Experiment würde eine im Voraus begrenzte und theoretisch gestellte Natur „befragt", ob und wie „sie sich meldet“ (Heidegger 2004a [1954]: 25).

Es lässt sich aufgrund der umfassenden editorischen Eingriffe Heideggers in das eigene Werk nicht abschließend klären, in welchen Etappen sich die heideggersche Faltung von Technik und Physik vollzog. Bereits in den Bremer Vorträgen, die er 1949 in Bremen und in schon leicht veränderter Form 1950 auf der Bühlerhöhe abhielt, finden sich zahlreiche Hinweise auf die Atomphysik. Besonders der Vortrag Das Ge-Stell enthält verschiedene Gegenwartsdiagnosen in Bezug auf nukleare Waffentechnologien (Heidegger 1994: 5, 27, 31, 43). Es ist allerdings unklar, aus welcher Zeit diese Bezüge stammen, denn die erste Wasserstoffbombenexplosion, auf die Heidegger Bezug nimmt, fand zum Beispiel erst 1952 statt. Wie bereits erwähnt, wurde Die Frage nach der Technik schon 1954 von Heidegger selbst veröffentlicht und markiert auch die philosophische Neuausrichtung Heideggers nach dem Zweiten Weltkrieg, während die Bremer Vorträge erst postum 1994 erschienen.

Das erste Treffen von Heidegger und Heisenberg fand im Übrigen bereits im Herbst 1935 in Heideggers Hütte in Todtnauberg statt. Heidegger sollte hier als Philosoph ein Gespräch zwischen dem Mediziner Viktor von Weizsäcker und Werner Heisenberg über die Rolle des Subjektes innerhalb der modernen Wissenschaften moderieren (von Weizsäcker 1977: 239 f.). Zeitgleich setzte Heideggers Beschäftigung mit der Quantenphysik ein, wie sie sich innerhalb der Einführung in die Metaphysik niedergeschlagen hat. Heideggers Auffassung der modernen Technik setzt also die heisenbergsche Situationsbeschreibung der Quantenmechanik voraus. Heideggers Satz, dass die neuzeitliche physikalische Theorie der Natur in ihrem Wesen technisch verfasst sei, ist auch als eine philosophische Interpretation des quantenmechanischen Experimental- und Naturbegriffs zu verstehen.

Erst 1953 und aufgrund der heisenbergschen Aussagen räumte Heidegger schließlich ein, dass die Quantenphysik mit einem gewandelten Kausalitätsbegriff operiere, was er nach seiner ersten Auseinandersetzung mit der 
Unbestimmtheitsrelation in den 1930er Jahren noch als transzendentale Verwechslung abgetan hatte (Heidegger 2009 [1953]: 174, 180). Vielleicht auch als Antwort auf Heisenbergs Aristoteles-Frage schlussfolgerte Heidegger in Die Frage nach der Technik, dass es angesichts der technologischen Situation keine causa efficiens oder causa finalis mehr gäbe, und damit auch die aristotelische Ursachenlehre nicht mehr gelte, genauso wenig wie die auf diese Weise gestellte Natur noch den Charakter des hervorbringenden Veranlassens, der poiesis zeige. Das Einzige, worauf diese Physik als Wissenschaft angewiesen bleibe, sei:

$[D] a ß$ sich die Natur in irgendeiner rechnerisch feststellbaren Weise meldet und als ein System von Informationen bestellbar bleibt [...]. Vermutlich schrumpft die Kausalität in ein herausgefordertes Melden gleichzeitig oder nacheinander sicherzustellender Bestände zusammen. Dem entspräche der Prozeß des zunehmenden Sichabfindens, den Heisenbergs Vortrag in eindrucksvoller Weise schilderte. (Heidegger 2004a [1954]: 26)

Heidegger übernahm Heisenbergs Verknüpfung von technischen Medien und naturwissenschaftlicher Evidenzproduktion und wendete sie zu einem eigenen Argument. Die Rückdatierung der modernen Technik auf die vorindustrielle Zeit, die Differenz zwischen „historisch rechnen“ und „geschichtlich denken“ (ebd.: 25) bedurfte offensichtlich der Rückbindung an Heisenbergs Überlegungen: Die Geschichte wachsender Abhängigkeit von Apparaturen wandele die Auffassung physikalischer Naturgesetzlichkeit, und damit auch der physikalischen Ontologie. Nicht die Natur sei Gegenstand der Physik, sondern das Verhältnis von Mensch und Natur, so schlussfolgerte er.

Heisenbergs Absatz über die kommende Atomtechnik enthält dann auch besonders viele Unterstreichungen durch Heidegger in dem annotierten Münchner Vortragsentwurf :

In der Atomtechnik schließlich handelt es sich ganz um die Ausnutzung von [solchen; Anm. MH] Naturkräften, zu denen jeder Zugang aus der Welt der natürlichen Erfahrung fehlt. Zwar wird uns vielleicht auch diese Technik schließlich ebenso geläufig werden wie dem modernen Menschen die Elektrotechnik, die aus seiner unmittelbaren Umwelt gar nicht mehr wegzudenken ist. [...] Dabei greift die Technik tief in das Verhältnis der Natur zum Menschen dadurch ein, daß sie seine Umwelt im großen Maßstab verwandelt und ihm damit den naturwissenschaftlichen Aspekt der Welt unablässig und unentrinnbar vor Augen führt. ${ }^{15}$

Der Verlust des objektiven Gegenstandes war aus Heideggers Sicht für die klassische Naturwissenschaft defizitär und die dabei entstehenden Technologien zielten vor allem auf "Steuerung und Sicherstellung“ des (Energie-) Bestands ab. Und dennoch, darauf bestand er, handele es sich dabei um Werke der aletheia, der Entbergung der physis: „Erschließen, umformen, speichern, verteilen, umschalten sind Weisen des Entbergens.", wie Heidegger es dann in Die Frage der Technik ausdrückte (Heidegger 2004a [1954]: 20). 
Durch die Auseinandersetzung mit Heisenberg erschien die moderne Technik, wie sie sich als Physik manifestiert, für Heidegger nicht nur als ein weiteres Erbe, sondern gar als Vollendung der neuzeitlichen Metaphysik. Für Heisenberg war dagegen die Situation innerhalb der naturwissenschaftlichen Grundlagen die Folge oder auch der Preis für den technischen Fortschritt innerhalb der experimentellen Physik, den man angesichts der neuen Möglichkeiten und Mächtigkeiten gerne in Kauf nahm. Die Technik war aus seiner Sicht der historische und ontologische Akteur, der Geschichte und Gegenstand der modernen Physik bedingte. Physikalische Wahrheit und Wirklichkeit seien dagegen sekundäre Ziele und letztendlich auch nur noch eine Frage der (Beobachtungs-)Technik. Die Quantenmechanik zwang Heidegger schließlich, die Physik aus dem Bereich einer nur im Ontischen operierenden Wissenschaft herauszuheben und sie an die Stelle der Metaphysik zu rücken. Im Atomzeitalter löste die Technik die Metaphysik als wissenschaftliches Verfahren ab. In Identität und Differenz von 1957 führte Heidegger dazu aus:

\begin{abstract}
Was jetzt ist, wird durch die Herrschaft des Wesens der modernen Technik geprägt, welche Herrschaft sich bereits auf allen Gebieten des Lebens durch vielfältige, benennbare Züge wie Funktionalisierung, Perfektion, Automatisation, Bürokratisierung, Information darstellt. So wie wir die Vorstellung vom Lebendigen Biologie nennen, kann die Darstellung und Ausformung des vom Wesen der Technik durchherrschten Seienden Technologie heißen. Der Ausdruck darf als Bezeichnung für die Metapyhsik des Atomzeitalters dienen (Heidegger 1990 [1957]: 42).
\end{abstract}

Auch innerhalb der heideggerschen Frage, was die Technik, das „Ge-stell“, wenn es denn eine Weise der Entbergung, ein Walten der Wahrheit ist, nun über das Sein, die physis ans Tageslicht bringt, scheint Heisenberg für Heidegger als eine Art Sprachrohr beziehungsweise Schriftführer des Gestells, als Stichwortgeber der Technik fungiert zu haben, wie seine Anstreichungen und Anmerkungen zum heisenbergschen Vortrag von 1953 belegen:

Wenn man versucht, von der Situation in der modernen Naturwissenschaft ausgehend sich zu den in Bewegung geratenen Fundamenten vorzutasten, so hat man den Eindruck, daß man die Verhältnisse vielleicht nicht allzu grob vereinfacht, wenn man sagt, daß zum ersten Mal im Laufe der Geschichte der Mensch auf dieser Erde nur noch sich selbst gegenüber steht [Anm. MH: Was ist der Mensch?], daß er keine anderen Partner oder Gegner mehr findet. Das gilt zunächst in einer ganz banalen Weise im Kampf des Menschen mit äußeren Gefahren. Früher war der Mensch durch wilde Tiere, durch Krankheiten, Hunger, Kälte und andere Naturgewalten bedroht, und in diesem Streit bedeutete jede Ausweitung der Technik eine Stärkung der Stellung des Menschen, also einen Fortschritt. In unserer Zeit, in der die Erde immer dichter besiedelt wird, kommt die Einschränkung der Lebensmöglichkeit und damit die Bedrohung in erster Linie von anderen Menschen, die auch ihr Recht auf die Güter der Erde geltend machen. In dieser Auseinandersetzung braucht die Erweiterung der Technik aber kein Fortschritt mehr zu sein. Der Satz, daß der Mensch nur noch sich selbst gegenüber stehe, gilt aber besonders im Zeitalter der Technik noch in einem viel weiteren Sinne. ${ }^{16}$ 
Der erste Teil des letzten Satzes wurde von Heidegger rot unterstrichen. In Die Frage nach der Technik findet sich die dazu passende Replik: „Indessen begegnet der Mensch heute in Wahrheit gerade nirgends mehr sich selber, d. h. seinem Wesen“ (Heidegger 2004a [1954]: 31).

Dass sich die Bausteine der Materie einer objektiven Festlegung in Raum und Zeit entziehen und der reale Gegenstand der Forschung nicht mehr durch die Teilchen selbst, sondern nur noch durch „unsere Kenntnis dieser Teilchen“ gebildet wird (Heisenberg 1956 [1953]: 18), stellte für Heidegger die Konsequenz experimenteller Technik dar.

In diesem Sinne übernahmen die Medien für Heidegger eine ontologische Funktion, indem sie das System aus sich meldender Natur und theoretischer Verifikation regeln. Dass am Grunde dieser Metaphysik mächtiger als zuvor der ontologische Riss zwischen Mensch und Welt, der verschränkende Schnitt zwischen Subjekt und Objekt und die Doppelnatur der physis operiert, darin sah er zugleich das „Entbergende“ und die Gefahr der Technik. In letzter Konsequenz bleibt das Verhältnis zwischen Mensch und Natur nach Heidegger nicht rationalisierbar und die Natur wird sich der Beherrschung durch die Technik immer entziehen. Heideggers Absage an die Kontrollierbarkeit der Technik hatte für Heisenberg allerdings keine praktischen Konsequenzen. Er war bereits 1953, wie Heidegger sehr wohl wusste, mit der Entwicklung des ersten nuklearen Forschungsreaktors in Deutschland beschäftigt (Heidegger 2005: 290). Für Heisenberg stand der unmittelbare ökonomische Nutzen der Atomtechnik im Vordergrund. So blieb der Briefwechsel eine philosophische, und wurde nicht zu einer physikalischen oder politischen Angelegenheit.

\section{Danksagung}

Ich bin dem Nachlassverwalter Heisenbergs Helmut Rechenberg zu großem Dank verpflichtet, mir Heideggers Briefe umgehend und unkompliziert zur Verfügung gestellt zu haben, ebenso wie Friedrich Kittler, Hermann Heidegger, Arnulf Heidegger und Ulrich von Bülow, die mich bei der Suche nach Heisenbergs Antworten und Heideggers schriftlichen Überlegungen zu Kausalität und Unbestimmtheitsrelation 2005 im Heidegger-Archiv sehr unterstützt haben.

\section{Anmerkungen}

1 Brief von Heidegger an Heisenberg, 18. März 1953, Nachlass Werner Heisenberg, MaxPlanck-Institut für Physik, München (im Folgenden abgekürzt als WHN).

2 Brief von Heidegger an Heisenberg, 9. Juni 1953, WHN. 
3 Brief von Heisenberg an Heidegger, 3. Oktober 1953 (Martin Heidegger-Nachlass, Konvolut A: Heidegger 1/B75; Nr. 75.7356, Deutsches Literaturarchiv Marbach (im Nachfolgenden abgekürzt als MHN).

4 Ebd., Hervorhebungen im Original.

5 Brief von Heidegger an Heisenberg, 8. November 1953, WHN.

6 Brief von Heisenberg an Heidegger, 3. Oktober 1953, MHN.

7 Brief von Heidegger an Heisenberg, 8. November 1953, WHN.

8 Brief von Heisenberg an Heidegger, 3. Oktober 1953, MHN.

9 Ebd.

10 Brief von Heidegger an Heisenberg, 8. November 1953, WHN.

11 Heidegger-Manuskript „Zu Heisenberg“, Hervorhebung im Original, MHN.

12 Heidegger-Manuskript „Fragen an Heisenberg“, ebd.

13 Heisenbergs Vortragsentwurf Das Naturbild der Physik mit Heideggers Unterstreichungen, ebd.

14 Ebd.

15 Ebd., Hervorhebung im Original.

16 Ebd.Hervorhebung im Original.

\section{Literatur}

Carson, Cathryn, 2010a. Science as Instrumental Reason. Heidegger, Habermas, Heisenberg, Continental Philosophical Review, 42, 483-509.

Carson, Cathryn, 2010b. Heisenberg in the Atomic Age. Cambridge, MA: Cambridge University Press.

Dämmerling, Christoph, 2003. Heidegger und die Frankfurter Schule. In: Dieter Thomä, Hg., Heidegger-Handbuch. Stuttgart/Weimar: Metzler Verlag, 361-369.

Habermas, Jürgen, 1953. Mit Heidegger gegen Heidegger denken, Frankfurter Allgemeine Zeitung, Nr. 170, 25. Juli 1953, Feuilleton: o. S.

Heidegger, Gertrud (Hg.), 2005. Mein liebes Seelchen. Briefe Martin Heideggers an seine Frau Elfriede. München: Deutsche Verlagsanstalt.

Heidegger, Martin, 1994. Bremer und Freiburger Vorträge (GA 79). Hg. von Petra Jäger. Frankfurt a. M.: Vittorio Klostermann.

Heidegger, Martin, 1990 [1957]. Identität und Differenz. 9. Aufl., Pfullingen: Neske.

Heidegger, Martin, 1998 [1953]. Einführung in die Metaphysik. 6. Aufl., hg. von Hermann Heidegger Tübingen: Max Niemeyer.

Heidegger, Martin, 2004a [1954]. Die Frage nach der Technik. In: Ders., Vorträge und Aufsätze. Stuttgart: Klett-Cotta, 9-40.

Heidegger, Martin, 2004b [1954]. Wissenschaft und Besinnung. In: Ders., Vorträge und Aufsätze. Stuttgart: Klett-Cotta, 41-66.

Heidegger, Martin, 2009 [1953]. Die Bedrohung der Wissenschaft. In: Ders., Leitgedanken zur Entstehung der Metaphysik, der neuzeitlichen Wissenschaft und der modernen Technik. Hg. von Claudius Strube. Frankfurt a. M.: Vittorio Klostermann, 157-190.

Heisenberg, Werner, 1949. Die gegenwärtigen Grundprobleme der Atomphysik. In: Ders., Wandlungen in den Grundlagen der Naturwissenschaft. Zürich: Hirzel, 89-101.

Heisenberg, Werner, 1956 [1953]. Das Naturbild der heutigen Physik. In: Bayerische Akademie der schönen Künste, Hg., Die Künste im technischen Zeitalter. 8. Aufl., Darmstadt: Wissenschaftliche Buchgesellschaft, 31-47.

Hempel, Hans-Peter, 1990. Natur und Geschichte. Der Jahrhundertdialog zwischen Heidegger und Heisenberg, Frankfurt a. M.: Anton Hain.

Hörl, Erich, 2004. Parmenideische Variationen. McCulloch, Heidegger und das kybernetische Ende der Philosophie. In: Claus Pias, Hg., Die Macy-Konferenzen, 1946-1953, Bd. 2., Berlin: Diaphanes.

Nagel, Ernest/Newman, James, 2001. Gödel's Proof. Hg. von Douglas R. Hofstadter, New York/ London: New York University Press.

Schiemann, Georg, 2008. Werner Heisenberg. München: C. H. Beck. 
von Weizsäcker, Carl Friedrich, 1977. Begegnungen in vier Jahrzehnten. In: Günther Neske, Hg., Erinnerung an Martin Heidegger. Pfullingen: Neske, 239-247.

\section{Christina Vagt}

Institut für Philosophie, Literatur-,

Wissenschafts- und Technikgeschichte,

Fachgebiet Literaturwissenschaft mit dem

Schwerpunkt Literatur und Wissenschaft

Technische Universität Berlin

Straße des 17. Juni 135

10623 Berlin

Deutschland

E-Mail: christina.vagt@tu-berlin.de 